\title{
Cirugía del colesteatoma y audición
}

\author{
Cholesteatoma surgery and audition
}

CarlosStott C1, Gonzalo Ortega F², Gustavo Bravo C1, Paul H. Délano $\mathbf{R}^{1,3}$.

\begin{abstract}
RESUMEN
Introducción: El colesteatoma es una entidad clínica que se presenta principalmente en edades medias de la vida. El tratamiento definitivo es quirúrgico, el cual apunta a erradicar la enfermedad, mientras que mantener o mejorar la audición es un objetivo secundario. Existen diversas técnicas quirúrgicas siendo la aticoantromastoidectomía con o sin preservación de la pared posterosuperior del conducto auditivo externo la más utilizada.

objetivos: Caracterizar a la población sometida a tratamiento quirúrgico del colesteatoma en el Hospital Clínico de la Universidad de Chile y determinar los cambios auditivos posoperatorios.

Material y método: Se realiza un estudio descriptivo retrospectivo en donde se analizan los procedimientos quirúrgicos entre los años 2007 y 2012. Se registran y analizan datos individuales, valores audiométricos pre y posoperatorios y tipo de cirugía realizada.

Resultados: Se incluyen 143 pacientes con promedio de edad de 41 años, siendo el $56 \%$ de sexo masculino. El 54,5\% fueron aticoantromastoidectomía; $21,6 \%$ aticoantrostomía; 16,7\% aticoantromastoidectomía más timpanoplastía IIIA y 6,7\% aticoantromastoidectomía más timpanoplastía IIIB. Los umbrales auditivos se mantuvieron en la aticoantrostomía, en la aticoantromastoidectomía y en la aticoantromastoidectomía con timpanoplastía tipo IIIB, mientras que la audición mejoró significativamente en las aticoantromastoidectomía con timpanoplastía tipo IIIA.

Conclusión: La técnica mayormente utilizada en el Hospital Clínico de la Universidad de Chile para el manejo del colesteatoma es la aticoantromastoidectomía con o sin preservación de la pared posterosuperior del conducto auditivo. Aquellos pacientes a los que se les asocia una timpanoplastía tipo IIIA presentan una mejoría auditiva significativa.

Palabras clave: Colesteatoma, umbrales auditivos, audición, aticoantrostomía, aticoantromastoidectomía, timpanoplastía.
\end{abstract}

\section{ABSTRACT}

Introduction: The cholesteatoma is a clinical entity that presents mostly in middle ages. The definitive treatment is surgery; which aims to eradicate the disease, while maintaining or improving hearing thresholds is a secondary goal. Several surgical techniques have

\footnotetext{
${ }^{1}$ Médico Otorrinolaringólogo, Servicio de Otorrinolaringología del Hospital Clínico Universidad de Chile.

${ }^{2}$ Interno de Medicina, Universidad de Chile.

${ }^{3}$ Programa de Fisiología y Biofísica, ICBM, Facultad de Medicina, Universidad de Chile.
} 
been described, being the tympanomastoidectomy with or without preservation of the postero-superior wall of the external auditory canal the most commonly used.

Aim: To characterize the population that underwent surgical treatment forcholesteatoma in the Clinical Hospital of the University of Chile and to determine the post-surgical hearing thresholds changes.

Methods: Retrospective and descriptive study of the surgical procedures carried out between the years 2007 and 2012. Individual data, pre and post-surgical treatment audiometric values and types of surgery are registered and analyzed.

Results: 143 patients were included with a 41 year age average, 56\% were males. $54,4 \%$ of surgeries were tympanomastoidectomy; $21,6 \%$ atticotomy; $16,7 \%$ tympanomastoidectomy with type IIIA tympanoplasty and 6,7\% tympanomastoidectomy with type IIIB tympanoplasty. Hearing thresholds were maintained in atticotomy, tympanomastoidectomy and tympanomastoidectomy with type IIIBtympanoplasty, while hearing thresholds improved in the tympanomastoidectomy with type IIIA tympanoplasty reconstruction.

Conclusion: The most used technique for cholesteatoma treatment in the Clinical Hospital of the University of Chile was tympanomastoidectomy with or without preservation of the posterosuperior wall of the external ear canal. Patients who had a type IIIA tympanoplastyimproved their hearing thresholds.

Key words: Cholesteatoma, hearing thresholds, audition, atticotomy, tympanomastoidectomy, tympanoplasty.

\section{INTRODUCCIÓN}

El colesteatoma es una lesión no neoplásica compuesta por un epitelio escamoso queratinizado de ubicación anómala. En el caso del oído se ubica en epitímpano, mastoides y en la cavidad del oído medio ${ }^{1,2}$. Presenta un patrón de crecimiento independiente capaz de reemplazar la mucosa, generar reabsorción ósea y tender a recurrir tras su extracción ${ }^{2}$.

El origen del colesteatoma puede ser de tipo congénito, en donde existen restos embrionarios de piel en presencia de un tímpano sano ${ }^{1}$; de tipo adquirido primario, el cual se origina a partir de un bolsillo de retracción ${ }^{1} 0$ de tipo adquirido secundario, el cual se origina a partir de una perforación timpánica marginal en la pars flácida 0 en la pars tensa ${ }^{3}$. Los adquiridos se consideran un tipo de otitis media crónica, siendo el adquirido primario el de mayor frecuencia ${ }^{1}$.

En términos generales este cuadro es poco frecuente, afectando a la población entre 3 a 70 años de edad, con una prevalencia estimada de $3 / 100.000$ en población pediátrica y 9/100.000 en adultos ${ }^{4,5}$

En el estudio histológico se observa un quiste benigno de células escamosas compuesto por dos elementos principales: la matriz o contenido, y la perimatriz o lámina propia ${ }^{4,6}$. La matriz se compone por epitelio escamoso queratinizado que da sostén a la estructura quística y la perimatriz es tejido de granulación. Este tejido de granulación puede producir enzimas proteolíticas y con esto generar destrucción ósea ${ }^{6,7}$.

La presentación clínica puede ser asintomática o presentarse como hipoacusia u otorrea. También puede debutar como una complicación; meningitis, abscesos intracraneales, laberintitis o parálisis facial ${ }^{1,4}$.

El tratamiento definitivo es únicamente quirúrgico donde el objetivo principal es lograr la erradicación de la enfermedad. Lograr una óptima protección del oído y mantener o mejorar la capacidad auditiva del paciente son objetivos secundarios del procedimiento ${ }^{8}$.

Existen diversas técnicas quirúrgicas, tales como la aticoantrostomía (AAT) con antroexclusión o como la aticoantromastoidectomía (AAM) con 0 sin preservación de la pared posterosuperior del conducto auditivo externo, también llamada cirugía

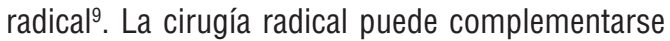
con una timpanoplastía; reconstrucción del oído medio con objetivo funcional ${ }^{10,11}$. Existen diversos tipos y subtipos de timpanoplastías, las cuales se basan en el daño del oído medio ${ }^{10}$. 
La timpanoplastía tipo III corresponde a la reconstrucción timpánica junto a la reparación de la cadena oscicular con apoyo en el estribo ${ }^{11}$. Esta se subdivide en timpanoplastía III. Asi la supraestructura del estribo está presente y en la cual el injerto se apoya directamente sobre la supraestructuradel estribo y en timpanoplastía tipo IIIB (T-IIIB), en donde la supraestructura del estribo está ausente quedando únicamente una platina móvil. Aquí se puede utilizar un injerto que permite la transmisión de las ondas sonoras desde el neotímpano a la platina del estribo ${ }^{11}$.

\section{OBJETIVO}

Describir y analizar el compromiso del colesteatoma según sexo, edad y oído afectado en el Hospital Clínico de la Universidad de Chile y analizar los resultados auditivos posquirúrgicos para el tratamiento del colesteatoma.

\section{MATERIAL Y MÉTODO}

Se realizó un estudio descriptivo retrospectivo en donde se recolectó y analizó la información obtenida de fichas clínicas de pacientes sometidos a cirugía para la erradicación del colesteatoma entre los años 2007 y 2012 en el Hospital Clínico de la Universidad de Chile. Se ingresó un total de 143 casos, de los cuales se registró información sobre edad, sexo, oído comprometido por colesteatoma, tipo de procedimiento quirúrgico realizado y promedio

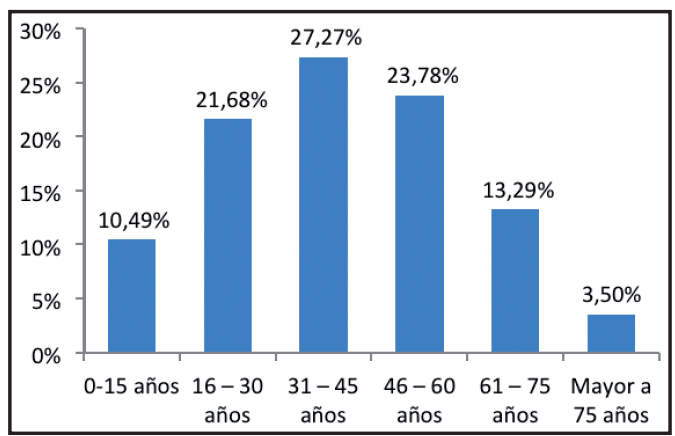

Figura 1. Distribución de los pacientes afectados por colesteatoma según rango etario. tonal puro (PTP) aéreo pre y poscirugía. Los datos obtenidos fueron divididos en 5 grupos: presencia de colesteatoma según sexo, rangos etarios, oído comprometido por colesteatoma, tipo de cirugía realizada y PTP aéreo pre y poscirugía según el procedimiento quirúrgico realizado.

Los datos se analizaron mediante estadística descriptiva y se calculó la significancia de los cambios de PTP aéreo pre y posoperatorios para cada tipo de cirugía otológica con la prueba de T de Student, considerando significativo un $p<0,05$.

\section{RESULTADOS}

Durante el periodo de estudio se registraron 143 procedimientos quirúrgicos para resolución de colesteatoma, constituidos por 80 hombres (56\%) y 63 mujeres (44\%). El promedio etario fue de 40,8 años, con rangos de edad entre los 3 y 82 años. Entre los 0 y 15 años se presentaron 15 casos (10\%), entre los 16 y 30 años 31 casos (22\%), entre los 31 y 45 años 39 casos (39\%), entre los 46 y 60 años 34 casos (24\%), entre los 61 y 75 años 19 casos (13\%) y sobre los 75 años 5 casos (3\%) (Figura 1).

Dentro del oído afectado, se observó que 67 pacientes tenían afectado el oído izquierdo (47\%), 66 pacientes tenían afectado el oído derecho (46\%) y 10 pacientes tenían afectación bilateral $(7 \%)$ (Figura 2).

Las cirugías sin intención funcional, aticoantrostomias (AAT) y aticoantromastoidectomía (AAM), fueron 109 (76\%); y las con intención funcional, aticoantromastoidectomías con timpa-

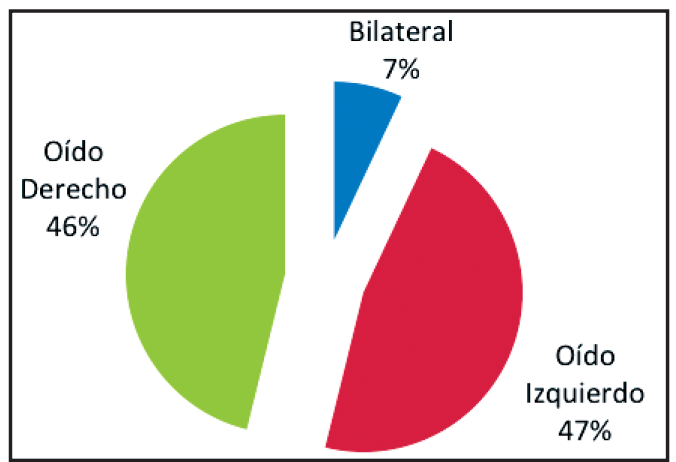

Figura 2. Distribución de los oídos afectados por colesteatoma. 
noplastía IIIA (AAM T-IIIA) 0 IIIB (AAM T-IIIB) fueron 34 (24\%) (Figura 3). Se realizaron un total de 31 AAT (22\%), 78 AAM (55\%), 24 AAM T-IIIA (17\%) y 10AAM T-IIIB (7\%) (Figura 4).

Los pacientes con datos completos para sus controles audiométricos de PTP aéreos pre y posoperatorios fueron 33, consistiendo en 8 AAT (26\% de las AAT), 16 AAM (20,5\% de las AAM), 6 AAM T-IIIA (25\% de las AAM T-IIIA) y 3 AAM T-IIIB (30\% de las AAM T-IIIB). Lamentablemente y debido a tratarse de un trabajo de tipo retrospectivo, el registro del control auditivo sólo se pudo obtener entre el $20 \%$ y $30 \%$ de las cirugías realizadas y sus conclusiones referentes a la audición representan sólo a 33 de 143 casos operados. Esto es muy relevante al analizar las timpanoplastías III A (6 casos) y especialmente la III B (3 casos).

Hubo una variación de los PTP aéreos en las AAT de 49,4 a 46,4 dB (diferencia 3,4; $p=0,63$ ) en las AAM de50 a $51 \mathrm{~dB}$ (diferencia $1 ; p=0,69$ ), en las AAM T-IIIA de 59,1 a 46,6dB (diferencia 12,5; $p=0,02$ ) y en las AAM T-IIIB de 73,6 a 72dB (diferencia 1,6; $p=0,66$ ) (Figura 5).

\section{DISCUSIÓN}

En la presente serie se observa una distribución similar entre ambos sexos y oídos afectados, concordando con la relación de hombres y mujeres de 1,2:1,0 de la población ${ }^{5,12}$. Similar a lo reportado en estudios previos, se observa una mayor proporción de pacientes con colesteatoma en edades medias de la vida ${ }^{4}$. Se obtuvo una baja prevalencia en edades

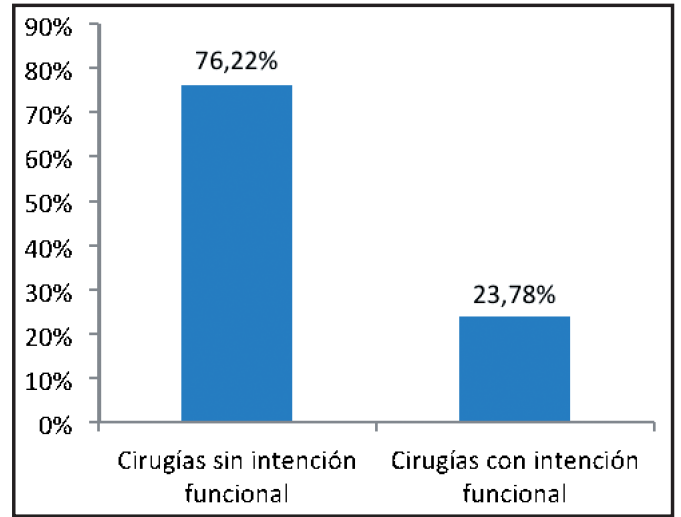

Figura 3. Distribución según cirugía con intención funcional o sin intención funcional.

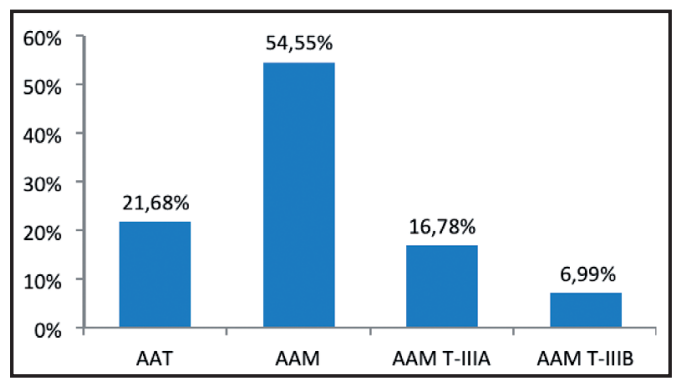

Figura 4. Distribución según tipo de cirugía realizada.

inferiores a los 15 años, edades de presentación del colesteatoma congénito, la cual es una entidad clínica infrecuente ${ }^{13}$.

Como sabemos, la resolución definitiva del colesteatoma es quirúrgica ${ }^{8}$. Se observó que en el Hospital Clínico de la Universidad de Chile la

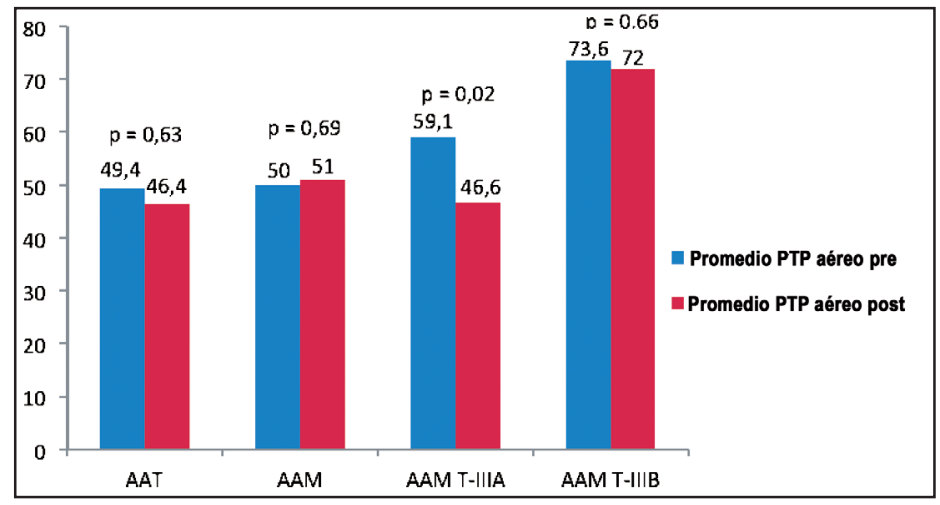

Figura 5. PTP aéreo (dB) pre y poscirugía del colesteatoma según tipo de intervención realizada. 
AAMes la técnica quirúrgica mayormente realizada, evidenciándose que además presenta una mantención de los umbrales auditivos posoperatoria. Esto es gracias a la eliminación de toda la fuente de inflamación y de hueso erosionado generada por el colesteatoma, manteniendo la anatomía del oído medio y de esta manera su funcionalidad².

Además se puede observar que la AAM T-IIIA presenta una mejoría significativa de la audición en el posoperatorio, lo cual se correlaciona con estudios nacionales ${ }^{11,14}$. Este resultado se explica por un daño menos avanzado de los mecanismos fisiológicos de la conducción sonora 11. En relación a las AAM T-IIIB, dado el número limitado de pacientes con audiometrías pre y posoperatorias ( $n=3)$, no es posible afirmar que la reconstrucción oscicular no sea útil para mejorar audición.

Si bien los resultados audiológicos, sólo representan $20 \%$ a $30 \%$ de los casos operados en esta serie, es importante resaltar que sólo la cirugía de aticoantromastoidectomía con timpanoplastia tipo IIIA tuvo mejorías de los umbrales auditivos. Además, dado que existen nuevas técnicas imagenológicas para el control posoperatorio de Ios colesteatomas, como la resonancia magnética cerebral con secuencia HASTE o PROPELLER ${ }^{15,16}$ pretendemos aumentar la proporción de pacientes operados incorporando técnicas de reconstrucción como la timpanoplastía tipo IIIA.

\section{CONCLUSIÓN}

Se obtuvieron resultados con una distribución epidemiológica de pacientes con colesteatoma similar a lo descrito en la literatura. En el Hospital Clínico de la Universidad de Chile se practica mayormente la aticoantromasoidectomía con o sin preservación de la pared posterior del conducto auditivo externo para el manejo del colesteatoma. Si bien los datos audiológicos representan una proporción reducida de nuestros pacientes, se puede observar que los pacientes sometidos a una cirugía radical conservan su audición, más aún, aquellos a los que se les realiza una cirugía radical con timpanoplastía tipo IIIA pueden llegar a mejorar sus umbrales audiométricos.

\section{BIBLIOGRAFÍA}

1. Cohen M, Callejas C, Salgado M. Fisiopatología del colesteatoma originado a partir de un bolsillo de retracción. Rev Otorrinolaringol Cir Cabeza Cuello 2006; 66: 39-46.

2. Stott C, Royer M, Ortúzar L, Pavon M. Resultados auditivos posaticoantrostomía con antroexclusión. Rev Otorrinolaringol Cir Cabeza Cuello 2007; 67: 129-34.

3. Quintero J, Abraham C, Hernández M, Meléndez L. Etiología del colesteatomaótico. Rev Cubana Pediatr 2011; 83: 393-404.

4. Olszewska E, Wagner M, Bernal-Sprekelsen M et AL. Etiopathogenesis of Cholesteatoma. Eur Arch Otorhinolaryngol 2004; 261: 6-24.

5. Leal A, Barbosa C, Quintaniliha F, Tavares J. Analysis of histopathological aspects in acquired middle ear cholesteatoma. Rev Bras Otorrinolaringol 2008; 74: 835-41.

6. Sade J, Halevy A. The etiology of bone destruction in chronic otitis media. J Laryngol Otol 1974; 88 : 139-43.

7. Chole R. Cellular and subcellular events of bone resorption in human and experimental cholesteatoma: the role of osteoclasts. Laryngoscope 1984; 94: 76-95.

8. Lacosta J, Infante J, Pisón F. Consideraciones sobre la cirugía funcional del colesteatoma. I. Técnicas cerradas. Acta Otorrínolaring Esp 1997; 48: 115-20.

9. Olaizola F. Nuestro criterio actual en la cirugía de lastimpanoplastias. Acta Otorrínolaring Esp 1989; 40: 219-22.

10. Fuentes A, Gaytán L, Hernández $S$. Timpanoplastía. Cinco años de experiencia en un hospital de enseñanza. Ann Med Asoc Med Hosp ABC 1996; 41: 6-9.

11. Nazar G, Bustos C, Larraguibel S, Larach F, IÑíguez R. Timpanoplastía tipo III. Rev Otorrinolaringol Cir Cabeza Cuello 2003; 63: 157-66.

12. Toro C, Naser A, Sanhueza C, Valdés C, Gormaz J, Molina V, Leslie A. Timpanoplastías en adultos en el Hospital Clínico de la Universidad de Chile: Revisión de 10 años. Rev Otorrinolaringol Cir Cabeza Cuello 2007; 67: 237-43.

13. Carmen L, Adiego I, Fernández R, Llorente E, Marín C, Hernández E. et al. Colesteatoma congénito de 
oído medio en la infancia. O.R.L. Aragón 2001; 4(1): 15-9.

14. Vergara V, Arancibia M, Corssen C, Cumsille M. Nuestro enfrentamiento a la patología de la otitis media crónica potencialmente peligrosa. Rev Otorrinolaringol Cir Cabeza Cuello 2003; 63: 95-9.

15. Alzérreca E, Garrido C, Zamorano R, Salas A, Delano PH. Resonancia magnética cerebral con secuencia difusión - HASTE en la evaluación clínica del colesteatoma. Rev Otorrinolaringol Cir Cabeza Cuello 2011; 71: 249-56.

16. Mateos-Fernández M, Mas-Estellés F, de PaulaVernetta C, Guzmán-Calvete A, Villanueva-Martí R, Morera-Pérez $C$. The role of diffusion-weighted magnetic resonance imaging in cholesteatoma diagnosis and follow-up. Study with the diffusion PROPELLER technique. Acta Otorrinolaringol Esp 2012; 63: 436-42.

Dirección: Dr. Gustavo Bravo

Departamento de Otorrinolaringología Hospital Clínico Universidad de Chile

Santos Dumont 999

E mail: guztab@gmail.com 